\title{
The small-angle performance of a dual-readout fiber calorimeter
}

\author{
A. Cardini ${ }^{c}$, M. Cascella ${ }^{d}$, S. Choi ${ }^{m}$, \\ D. De Pedis ${ }^{g}$, R. Ferrari ${ }^{h}$, S. Franchino ${ }^{i}$, \\ G. Gaudio ${ }^{h}$, S. Ha ${ }^{m}$, J. Hauptman ${ }^{j}$, \\ L. La Rotonda ${ }^{k}$, S. Lee ${ }^{m, n}$, F. Li ${ }^{j}$, M. Livan ${ }^{f}$, \\ E. Meoni ${ }^{l}$, F. Scuri ${ }^{b}$, A. Sill ${ }^{a}$, and R. Wigmans ${ }^{a, 1}$ \\ ${ }^{a}$ Texas Tech University, Lubbock (TX), USA \\ ${ }^{b}$ INFN Sezione di Pisa, Italy \\ ${ }^{c}$ INFN Sezione di Cagliari, Monserrato (CA), Italy \\ ${ }^{d}$ Dipartimento di Fisica, Università di Salento, and INFN Sezione di Lecce, Italy \\ ${ }^{f}$ INFN Sezione di Pavia and Dipartimento di Fisica, Università di Pavia, Italy \\ ${ }^{g}$ INFN Sezione di Roma, Italy \\ ${ }^{h}$ INFN Sezione di Pavia, Italy \\ ${ }^{i}$ CERN, Genève, Switzerland \\ ${ }^{j}$ Iowa State University, Ames (IA), USA \\ ${ }^{k}$ Dipartimento di Fisica, Università della Calabria, and INFN Cosenza, Italy \\ ${ }^{l}$ Tufts University, Medford (MA), USA \\ ${ }^{m}$ Korea University, Seoul, Korea \\ ${ }^{n}$ Kyungpook National University, Daegu, Korea
}

\begin{abstract}
The performance of the RD52 dual-readout calorimeter is measured for very small angles of incidence between the $20 \mathrm{GeV}$ electron beam particles and the direction of the fibers that form the active elements of this calorimeter. The calorimeter response is observed to be independent of the angle of incidence for both the scintillating and the Čerenkov fibers, whereas significant differences are found between the angular dependence of the energy resolution measured with these two types of fibers. The experimental results are on crucial points at variance with the predictions of GEANT4 Monte Carlo simulations.
\end{abstract}

PACS: 29.40.Ka, 29.40.Mc, 29.40.Vj

Key words: Dual-readout calorimetry, Čerenkov light, optical fibers

$\overline{1}$ Corresponding author. Email wigmans@ttu.edu, fax (+1) 806 742-1182.

Preprint submitted to Nuclear Instruments and Methods A

29 October 2015

(C) 2015. This manuscript version is made available under the Elsevier user license http://www.elsevier.com/open-access/userlicense/1.0/ 


\section{Introduction}

Sampling calorimeters based on large numbers of optical fibers embedded in a metal absorber structure offer some distinct advantages compared to other detectors of high-energy particles. Since the fibers act at the same time as the active medium in which the signals are produced and as a wave guide transporting the signals to the outside world, it is posssible to construct hermetic detector structures, which is very important in modern colliding-beam experiments. Also, the very frequent shower sampling allowed by a fiber configuration strongly reduces the effects of sampling fluctuations. Such fluctuations tend to dominate the energy resolution of electromagnetic sampling calorimeters. Several particle physics experiments have taken advantage of these features, e.g., CHORUS [1], KLOE [2], DELPHI [3], WA89 [4], H1 and CMS [5].

In dual-readout calorimeters, two different types of signals are produced by the showering particles. These two types of signals, which represent the total energy deposit by ionization $(d E / d x)$ and the Čerenkov light produced by the relativistic shower products, provide complementary information, which makes it for example possible to determine the electromagnetic fraction of each hadronic shower. The fluctuations in that fraction typically dominate the hadronic energy resolution of calorimeters, and dual-readout calorimeters thus offer the possibility to eliminate the effects of these fluctuations and obtain excellent hadronic performance [6,7].

In the calorimeter discussed in this paper, signals are generated in scintillating fibers, which measure the deposited energy, and in clear plastic fibers, which measure the relativistic shower particles, by means of the Čerenkov light generated by these. A large number of such fibers are embedded in a copper absorber structure. This detector is longitudinally unsegmented, the fibers are oriented in approximately the same direction as the particles to be detected. In previous papers, we have focused on the electromagnetic performance of this detector [8] and on its capability to identify the particles developing showers in it [9]. In this paper, we investigate its performance as a function of the angle of incidence of the showering particles. This study was inspired by the results of Monte Carlo simulations, which suggested anomalous effects when electrons or photons enter the calorimeter almost parallel to the optical fibers [10]. These effects derive from the fact that the early component of em showers (i.e., before the shower maximum is reached) is extremely collimated. When an electron enters this calorimeter parallel to the fibers, the signal from the early shower component strongly depends on the impact point, i.e., inside a fiber or in between fibers. This effect leads to a deterioration of the em energy resolution and may also affect the calorimeter response ${ }^{2}$.

In Section 2, the instruments and the experimental setup in which the measurements

2 Following the convention introduced in [11], we define the calorimeter response in this paper as the average calorimeter signal per unit deposited energy. In this convention, a linear calorimeter thus has a constant response, and a compensating calorimeter has the same response for electrons and hadrons. 
were carried out are described, as well as the calibration and data analysis methods that were used. Experimental results are presented in Section 3. In the concluding Section 4, we discuss these results and their implications.

\section{Equipment and measurements}

\subsection{Detectors and beam line}

The measurements described in this paper were performed in the H8 beam line of the Super Proton Synchrotron at CERN. For these particular studies, we used a beam of $20 \mathrm{GeV}$ electrons, which were steered into a module of the RD52 fiber calorimeter. A system of auxiliary detectors, described below, was used to select the beam particles that entered the calorimeter in a well defined, small area.

The calorimeter module used for the studies described in this paper consisted of a copper absorber matrix, $2.5 \mathrm{~m}$ long $\left(10 \lambda_{\text {int }}\right)$, with a cross section of $9.2 \times 9.2 \mathrm{~cm}^{2}$. It was subdivided into four towers $\left(4.6 \times 4.6 \times 250 \mathrm{~cm}^{3}\right)$, and each tower contained 1024 plastic optical fibers (diameter $1.0 \mathrm{~mm}$, equal numbers of scintillating and clear plastic fibers $)^{3}$. Each tower produced two signals, a scintillation signal and a Čerenkov signal, which were detected by separate PMTs ${ }^{4}$. For this reason, this type of detector is also known as a DREAM (Dual-REAdout Method) calorimeter. The fiducial mass of the calorimeter module was $\sim 120 \mathrm{~kg}$, it contained $45 \%$ fibers by volume and the sampling fraction for minimum ionizing particles, both for the scintillation and for the Čerenkov sampling structure, was $4.6 \%$. The effective radiation length and Moliere radius were both about $25 \mathrm{~mm}$. Figure 1a shows this module while it was under construction; details of the fiber structure, as well a front view of the finished product, are shown in Figure 1b, where the scintillating and Čerenkov fibers are indicated by $S$ and $\check{C}$, respectively.

The experimental setup contained also a number of auxiliary detectors, which were intended to determine the identity of individual beam particles, and to measure their trajectory. Figure 1c shows a schematic overview of the beam line, in which the positions of these auxiliary counters are indicated:

- Two small scintillation counters provided the signals that were used to trigger the data acquisition system. These Trigger Counters $\left(T_{1}, T_{2}\right)$ were $2.5 \mathrm{~mm}$ thick, and the area of overlap was $4 \times 4 \mathrm{~cm}^{2}$. Downstream from these counters, a third scintillation counter $\left(T_{H}\right)$ was installed. The latter had a hole with a radius of $10 \mathrm{~mm}$ in it. A (anti-)coincidence between the logic signals from these counters

\footnotetext{
$\overline{3}$ The scintillating fibers were of the type SCSF-78 (polystyrene core), produced by Kuraray. Their numerical aperture was 0.55 and the cladding thickness $20 \mu \mathrm{m}$. The Čerenkov raray. Their numerical aperture was 0.55 and the cladding thickness $20 \mu \mathrm{m}$. The Cerenkov
light was generated in PMMA based SK40 fibers, produced by Mitsubishi. Their numerical aperture was 0.50 , and the thickness of the cladding $10 \mu \mathrm{m}$. 4 Hamamatsu R8900, 10-stage.
} 

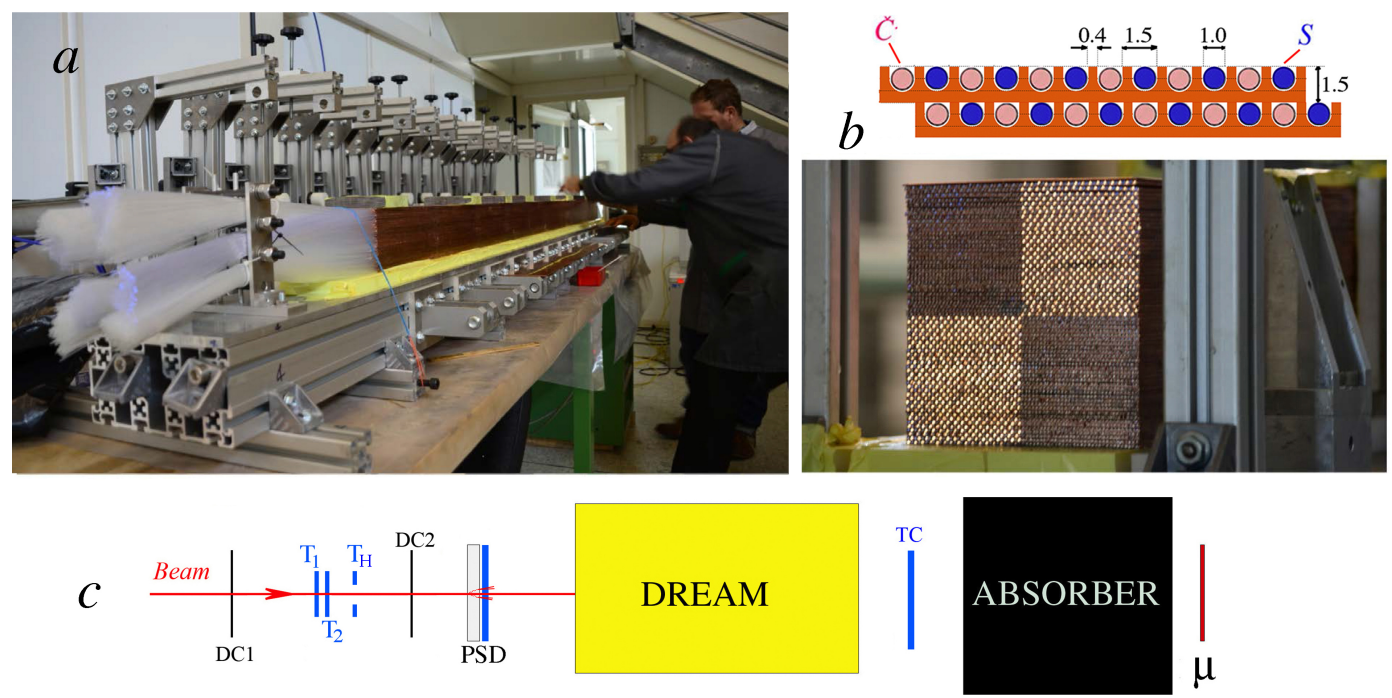

Fig. 1. The calorimeter module used for these studies $(a, b)$. Schematic overview of the arrangement of the auxiliary detectors that were used to define the beam spot and to identify the individual beam particles ( $c$, not to scale). See text for details.

provided the trigger $\left(T_{1} \cdot T_{2} \cdot \overline{T_{H}}\right)$.

- The trajectories of individual beam particles could be reconstructed with the information provided by two small drift chambers (DC1, DC2), which were installed upstream and downstream of the trigger counters. This system made it possible to determine the location of the impact point of the $20 \mathrm{GeV}$ beam particles at the calorimeter surface with a precision of about $2 \mathrm{~mm}$.

- About $80 \mathrm{~cm}$ upstream of the calorimeter, a preshower detector (PSD) provided signals that could be used to remove pions and muons contaminating the electron beams. This PSD consisted of a $5 \mathrm{~mm}$ thick lead plate, followed by a 5 $\mathrm{mm}$ thick plastic scintillator. Electrons started developing showers in this device, while muons and hadrons typically produced a signal characteristic of a minimum ionizing particle (mip) in the scintillator plate.

- Downstream of the calorimeter (DREAM), a tail catcher (TC) also served to identify pions and muons, since the electron showers were typically fully contained in the calorimeter. This tail catcher consisted of a simple $20 \times 20 \mathrm{~cm}^{2}$ scintillation counter.

- Further downstream of the calorimeter, behind an additional $8 \lambda_{\text {int }}$ worth of absorber, a $50 \times 50 \mathrm{~cm}^{2}$ scintillation counter $(\mu)$ served to identify muons that contaminated the particle beam.

5 We thank Michael Jeckel and Ilias Efthymiopoulos for making these modifications to our equipment. 


\subsection{Data acquisition}

Low-loss cables with a 15-mm diameter were used to transport the signals from the trigger counters to the counting room. The signal speed in these cables was measured to be $0.78 c$. The calorimeter signals, as well as the signals from the auxiliary counters that needed to be digitized (PSD, tail catcher, muon counter) were transported through RG-58 cables with (for timing purposes) appropriate lengths to the counting room.

There, the signals to be digitized were fed into charge ADCs. The signals from the wire chambers were fed into TDCs. The time information could be converted into $(x, y)$ coordinates of the point where the beam particle traversed the chamber.

The data acquisition system used VME electronics. Two VME crates hosted all the needed readout and control boards. The signals from the calorimeter channels and the auxiliary detectors were integrated and digitized with a sensitivity of 100 fC/count, on 12-bit QDC V792 CAEN modules. The timing information of the tracking chambers was recorded with 1 ns resolution in a 16-bit 16-channel CAEN V775N TDC. Our readout scheme optimized the CPU utilization and the data taking efficiency using the bunch structure of the 42-second SPS accelerator cycle, during which period beam particles were provided to our experiment by means of two extractions with a duration of 4.8 seconds each.

\subsection{Experimental data, calibration and analysis methods}

The measurements described in this paper were performed in the $\mathrm{H} 8$ beam of the CERN Super Proton Synchrotron. Beams enriched in electrons were derived from a secondary beam of positively charged particles, by sending these particles through a $5 \mathrm{~mm}$ thick lead radiator. In practice, only the electron component of the secondary beam lost a substantial energy fraction passing through this material, and electrons ${ }^{6}$ of the desired momentum $(20 \mathrm{GeV})$ were selected with properly tuned downstream bending magnets and collimators.

The measurements were performed by steering the beams into a region near the geometrical center of the module. Typically, for each run 20000 events were collected. In each run, $10 \%$ randomly triggered events provided pedestal information. For each event, the ADC information of all calorimeter towers was recorded, as well as the ADC data from the auxiliary detectors (muon counter, PSD, tail catcher), and the TDC data from the wire chambers.

Off-line, the beam chamber information could be used to select events that entered the calorimeter in a small region (typically with a radius $<5 \mathrm{~mm}$ ) located near

6 The selected particles were positively charged anti-electrons (positrons). However, we systematically refer to these particles as electrons in this paper. 


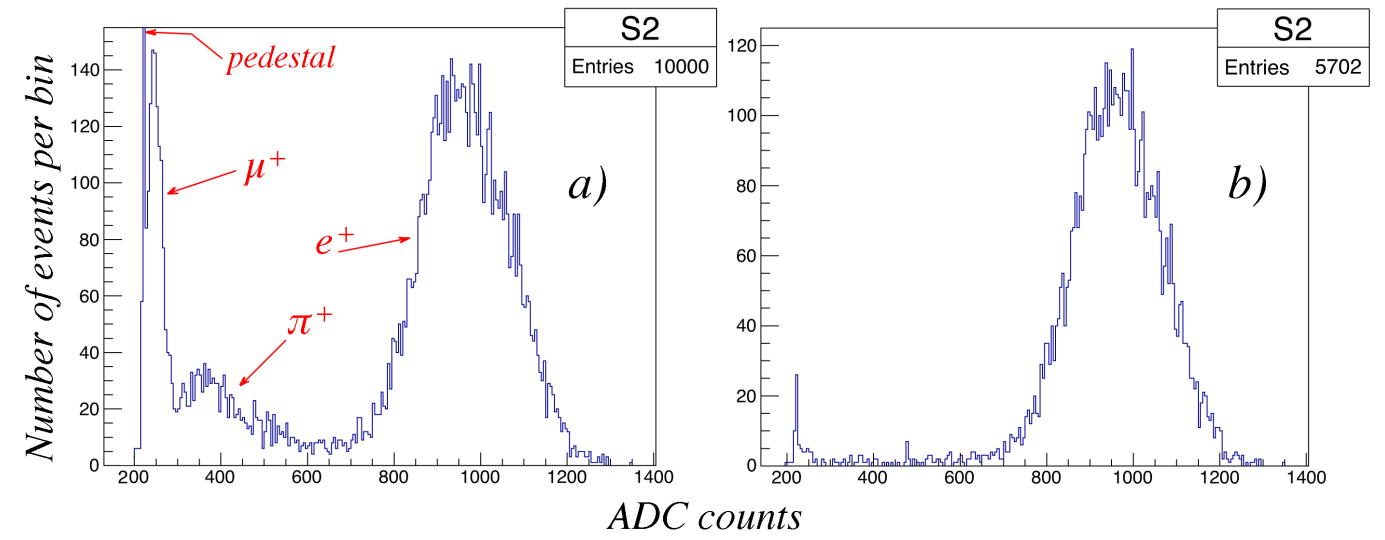

its geometric center. The information provided by the auxiliary detectors was used to identify each event either as an electron, a muon or a pion. It turned out that the $20 \mathrm{GeV}$ beam consisted predominantly (> $50 \%$ ) of electrons. However, there was also a substantial contamination by muons and pions. Since the beam profiles of the various types of beam particles differed, the precise fraction of electrons depended on the chosen size of the beam spot: the smaller the beam spot, the purer the electron sample.

The analyses described in Section 3 were performed on purified event samples of electrons. To that end, the following cuts were applied. Electrons were identified as particles that produced a signal in the PSD that was larger than $\sim 200$ ADC counts above pedestal, which corresponds to the combined signals produced by 2 minimum ionizing particles (mips) traversing this detector. Additional requirements were that no signals incompatible with electronic noise were produced in the tail catcher and the muon counter. The effects of these cuts on the scintillation signals from one of the four calorimeter towers is illustrated in Figure 2. In order to further limit the event sample to electrons that deposited (most of) their energy in the calorimeter, the total scintillation signal of the calorimeter was also required to be larger than $15 \mathrm{GeV}$.

Fig. 2. The scintillation signal distribution in one of the calorimeter towers before $(a)$ and after $(b)$ applying the cuts intended to eliminate the contaminating muons and pions from the $20 \mathrm{GeV}$ particle beam.

The electrons deposited typically $\sim 1 \%$ of their energy in the preshower detector. The effects of that on the calorimeter signals were studied in the present analyses. This was possible since all angular scans were performed separately with and without the PSD in the beam line. In the latter case, the event samples inevitably contained some fraction of pions (typically $\sim 10 \%$ ). Inspired by the results of an earlier study [8], we found that this hadron contamination could be effectively reduced by using the ratio of the two types of calorimeter signals, $C / S$. However, it also turned out that by applying such a cut, a bias was introduced which improved the em energy resolution in the scintillation channel significantly. We come back to this issue in Section 4. For this reason, the $C / S$ calorimeter signal ratio was not used in the analyses described in Section 3, and the effects of the PSD on the energy 
resolution were studied by using the PSD just as a passive absorber, i.e., without making use of the signals it generated. For both data sets, we used exactly the same procedure to purify the electron event samples, and this procedure was exclusively based on the auxiliary detectors, not on the calorimeter data.

In this paper, we call the angle between the particle trajectory and the fiber direction $\theta$ in the vertical plane and $\phi$ in the horizontal plane. The detailed angular scans of which the results are the topic of this paper were possible because the calorimeter was mounted on a table that could be rotated in the horizontal plane; the azimuth angle $\phi$ could be varied with a precision and reproducibility of about 1 milliradian. In the vertical plane, a deviation of the angle $\theta$ was achieved by tilting the calorimeter module. Angular $\phi$ scans were carried out for two values of the tilt angle: $\theta=1^{\circ}$ and $\theta=2^{\circ}$.

The calibration of the calorimeter towers was performed by steering the $20 \mathrm{GeV}$ electron beam into the centers of each of the four calorimeter towers. The PSD was installed in the beam line during these measurements, allowing the selection of a pure sample of beam electrons for this purpose. In an analysis described elsewhere [8], we found that the electrons deposited, on average, $85 \%$ of their energy in the hit tower, so that the average signal corresponded to an energy deposit of $\sim 17 \mathrm{GeV}$. The calibration of the signals, in terms of GeV per ADC count, was performed in two steps. First, the average signals from the four towers were equalized, in terms of GeVs. Second, an overall multiplication factor was determined from the requirement that the total calorimeter signal, for electrons entering the calorimeter in the central region, parallel to the fibers, equals the beam energy of $20 \mathrm{GeV}$. This procedure was applied separately for the scintillation and the Čerenkov signals. The calibration was performed at $\left(\theta=1^{\circ}, \phi=0^{\circ}\right)$ with the PSD installed in the beam line. It was repeated several times during the beam tests (which lasted one week) and found to be stable within $1 \%$.

When the table on which the detector was installed was rotated, the impact point of the beam particles shifted. For a rotation from $\phi=0$ to the maximum angle used in these studies $\left(\phi=5^{\circ}\right)$, this shift amounted to $120 \mathrm{~mm}$. For each angle of incidence $\phi$, the horizontal displacement was chosen such that the shower maximum was always located in the same area (at a depth of $\sim 15 \mathrm{~cm}$ ) inside the calorimeter. This was done to minimize any systematic effects caused by fiber-to-fiber response differences.

\subsection{Why $20 \mathrm{GeV}$ electrons?}

The electromagnetic energy resolution, $\sigma / E$, of a calorimeter such as this one typically consists of the following components:

- A stochastic term, which is determined by sampling fluctuations and fluctuations in the number of photoelectrons 
- A noise term, caused by pedestal fluctuations

- A term resulting from shower leakage fluctuations

- An energy independent (or constant) term, caused by instrumental effects, such as fiber-to-fiber response differences, position dependence of the quantum efficiency of the PMTs, etc.

The first three terms depend on the energy of the showering particles. Leakage fluctuations depend, in addition, also on the type of leakage (lateral, longitudinal, albedo). Only the contribution of lateral leakage fluctuations to the em energy resolution is a consideration for our detector. They depend on the shower energy as $x \% / \sqrt[4]{E}[11]$, where $x$ is the average leakage percentage ( $\sim 4 \%$ for small angles of incidence). The contribution of the stochastic term to the energy resolution scales with $1 / \sqrt{E}$, that of the noise term with $1 / E$, while the contribution of the constant term does not change with the particle energy. As a result, the energy resolution of the calorimeter is dominated by the noise term at very low energies and by the constant term at the high end of the energy spectrum.

Our studies were inspired by the possible effects of changes in the sampling characteristics of the calorimeter for very small angles of incidence. Therefore, we chose a particle energy for which the energy resolution is dominated by the contribution of the stochastic term. This consideration led to the choice of $20 \mathrm{GeV}$ electrons. At this energy, the contributions of the four terms mentioned above to the relative em energy resolution were estimated to be $4 \%,<1 \%, 2-3 \%$ and $<1 \%$, repectively, for the scintillation signals, and 5\%, $<1 \%, 1-2 \%$ and $<1 \%$, for the Čerenkov signals. The contribution of energy independent effects was minimized by using a small beam spot and by steering the beam such that the location of the shower maximum was independent of the angle of incidence (see section 2.3).

Based on measurements with the original DREAM detector [12], we estimate the average shower containment at $96 \%$, given the effective radial size of 2.1 Moliere radii. This value is for the angle at which the module was calibrated. At larger angles, the shower maximum (located at a depth of $\sim 15 \mathrm{~cm}$ ) stays at the same spot, but the shower containment is probably a bit smaller because of side leakage from the tail. The containment is better for the Čerenkov signals.

In the following, the measured energy resolution, $\sigma / E$, has been determined on the basis of Gaussian fits to the pulse height spectra, over a range spanning \pm 2 standard deviations around the mean value. Examples of such fits are shown in Figures 4 and 14.

\section{Experimental results}

\subsection{The angular dependence of the calorimeter response}

Angular scans were performed for two values of the tilt angle: $\theta=1^{\circ}$ and $2^{\circ}$. Data were collected for $20 \mathrm{GeV}$ electrons entering the calorimeter at azimuth angles 
$\phi$ ranging from $-5^{\circ}$ to $+5^{\circ}$. In order to check possible effects of the upstream preshower detector, all measurements were carried out with and without the PSD in place. The impact point of the beam was chosen near the geometric center of the calorimeter, and events were selected that entered the calorimeter within a circle of radius $4 \mathrm{~mm}$ around that point (Figure 3 ). In the following, we only show results for the angular range from $-4^{\circ}$ to $+3^{\circ}$.

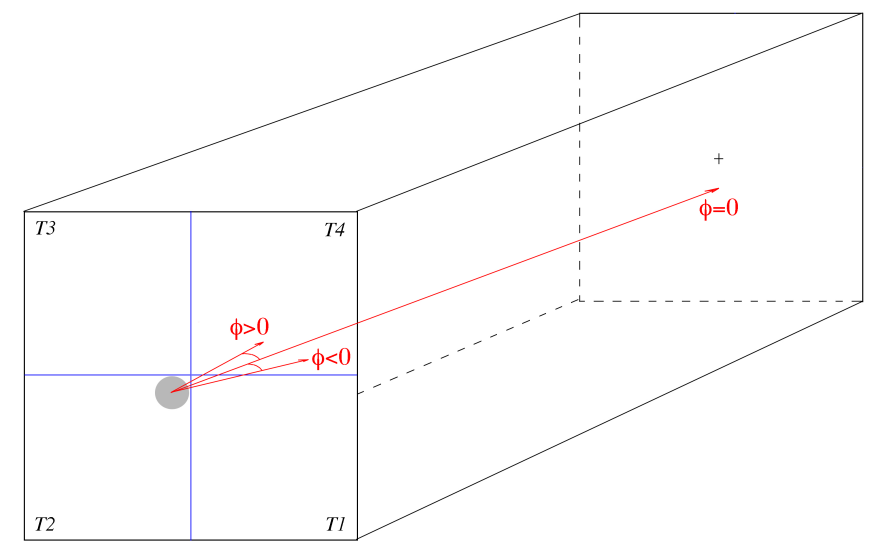

Fig. 3. Schematic view of the calorimeter, showing the four towers into which it is divided. The beam spot is indicated by the shaded area. The direction of the beam line is indicated for different angles of incidence of the beam particles.

It turned out that at larger angles, lateral leakage effects and the fact that different fibers contributed significantly to the signals started to play a role, and these effects were not of interest for our present studies ${ }^{7}$.

Figure 4 shows typical signal distributions recorded for the Čerenkov signals, with the preshower detector in (Figure 4a) or out of the beamline (Figure 4b). The figure illustrates not only the capability of the preshower detector to remove the pion contamination by means of the signal it generates, but also the effects of this upstream absorber on the calorimeter signals. When this aborber is removed, the calorimeter signals increase, on average, a little bit (about $2 \%$ in this example). In addition, the calorimeter's energy resolution improves as a result of the absence of fluctuations in the energy fraction lost in the upstream absorber.

In Figure 5, the measured calorimeter response is shown as a function of the azimuth angle of incidence $\phi$. Results are given separately for the scintillation and the Čerenkov signals, for a tilt angle $\theta=1^{\circ}$, with the preshower detector in the beam line (PSD IN). It turns out that the calorimeter signal varied by less than one percent for angles of incidence ranging from $-4^{\circ}$ to $+4^{\circ}$, both for the scintillation and the Čerenkov channels. In fact, the distribution of the calorimeter response obtained for the 21 different angles for which it was measured varied only by $0.34 \%$

7 Since the beam spot was chosen slightly off-center, leakage effects also affected the results at $\phi=+4^{\circ}$. The data point $\left(\theta=1^{\circ}, \phi=3^{\circ}\right.$, PSD IN) was forgotten in the data taking procedures, and is thus missing in Figures 5,7,8. 


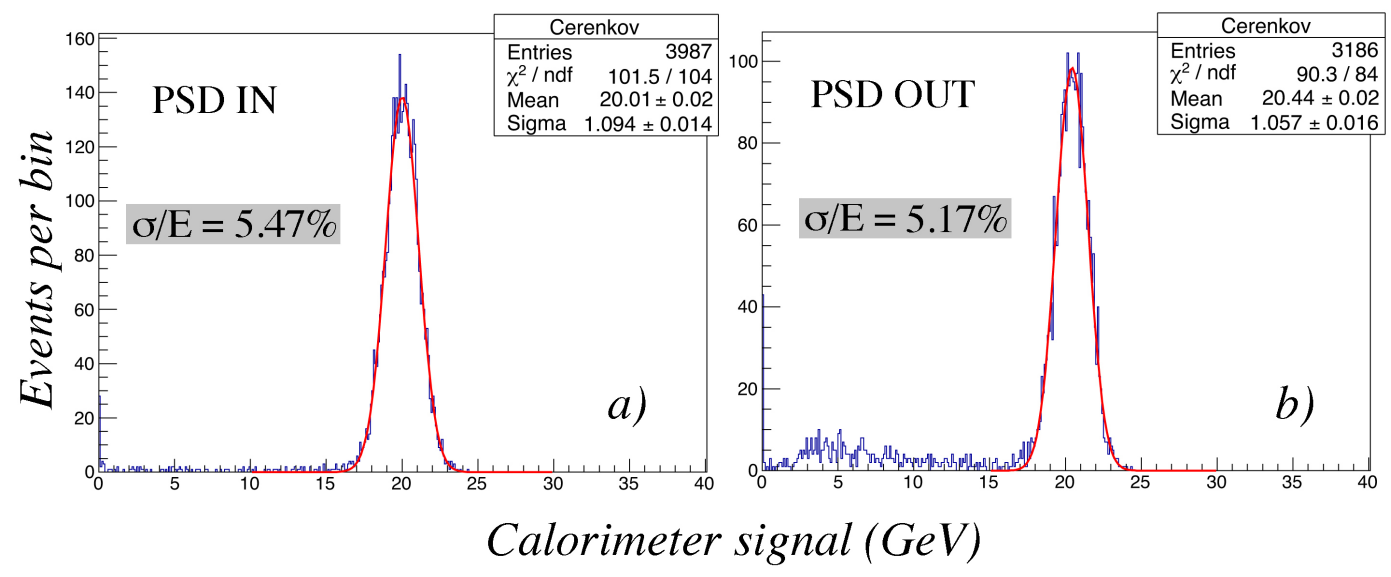

Fig. 4. Typical calorimeter signal distributions recorded with the preshower detector in $(a)$ or out $(b)$ of the beam line. These distributions were obtained for the Čerenkov signals, at angles $\phi=-0.2^{\circ}$ and $\theta=2^{\circ}$.

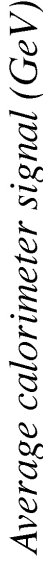

and $0.35 \%$ for the two types of signals $\left(\sigma_{\mathrm{rms}}\right)$. We measured the response variations also for the other sets of measurements, i.e., with the PSD IN or OUT, and for tilt angles $\theta$ of $1^{\circ}$ or $2^{\circ}$. The results are summarized in Table 1 .

For all these configurations, the response variations were measured to be smaller than $1 \%$, represented by the shaded area around the average signal shown in Figure 5. No experimental evidence was found for the specific angular response dependence predicted by GEANT4 Monte Carlo simulations[10] in any of these measurement series.

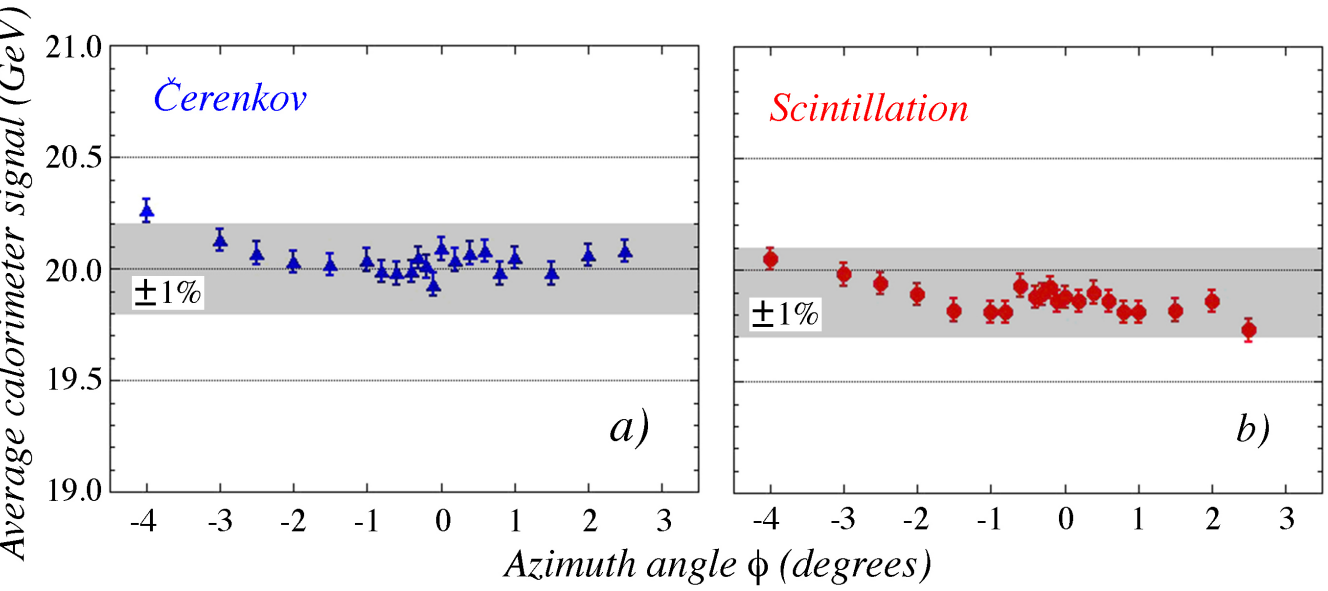

Fig. 5. The average calorimeter signals for $20 \mathrm{GeV}$ electrons in the Čerenkov (a) and the scintillation $(b)$ channels, as a function of the azimuth angle of incidence $(\phi)$ of the beam particles. The tilt angle $\theta$ was $1^{\circ}$, and the PSD was in the beam line. The shaded area represents a variation of $\pm 1 \%$ with respect to the average signal. 
Table 1

Variation of the calorimeter response to $20 \mathrm{GeV}$ electrons with the azimuth angle of incidence $\phi$ ranging from $-4^{\circ}$ to $+4^{\circ}\left(\sigma_{\text {rms }}\right.$ expressed as a percentage of the average calorimeter signal).

\begin{tabular}{|lccc|}
\hline Signal type & Preshower Detector & Tilt angle $\theta$ & Response variation \\
\hline Čerenkov & IN & $1^{\circ}$ & $0.34 \%$ \\
Scintillation & IN & $1^{\circ}$ & $0.35 \%$ \\
Čerenkov & OUT & $1^{\circ}$ & $0.56 \%$ \\
Scintillation & OUT & $1^{\circ}$ & $0.81 \%$ \\
Čerenkov & IN & $2^{\circ}$ & $0.33 \%$ \\
Scintillation & IN & $2^{\circ}$ & $0.44 \%$ \\
Čerenkov & OUT & $2^{\circ}$ & $0.36 \%$ \\
Scintillation & OUT & $2^{\circ}$ & $0.85 \%$ \\
\hline
\end{tabular}

\subsection{The angular dependence of the energy resolution}

\subsubsection{The difference between the scintillation and Čerenkov signals}

The energy resolutions measured for the $20 \mathrm{GeV}$ electrons showering in the calorimeter are shown in Figure 6 as a function of the azimuth angle of incidence $\phi$, separately for the scintillation and the Čerenkov signals. The tilt angle $\theta$ was $1^{\circ}$ and the preshower detector was out of the beam line for these particular results. The figure shows remarkable differences between the two types of signals. Not only is the energy resolution significantly worse for the scintillation signals, for all angles in this range, but the resolution measured for the scintillation signals also depends strongly on the angle of incidence, unlike the resolution measured with the Čerenkov signals.

These results are consistent with earlier observations that the energy resolution at a fixed angle of incidence $\left(\phi=1.5^{\circ}, \theta=1.0^{\circ}\right)$ tends to become better for the Cerenkov signals than for the scintillation ones as the energy of the electron beam increases [8]. Since the sampling structure is the same for both types of fibers, and the light yield considerably larger (and event-to-event fluctuations in the number of scintillation photoelectrons thus correspondingly smaller), one would naively expect to measure better energy resolutions for the scintillation signals. The fact that the opposite effect is experimentally observed is a consequence of the extremely collimated nature of the em showers in the early stage of the shower development, before the shower maximum is reached, i.e., in the first $10 \mathrm{~cm}$ of this particular calorimeter.

Neighboring fibers of the same type are separated by 2 - $3 \mathrm{~mm}$ (edge-to-edge, see Figure 1b) and that distance is of the same order as the shower width in this early 


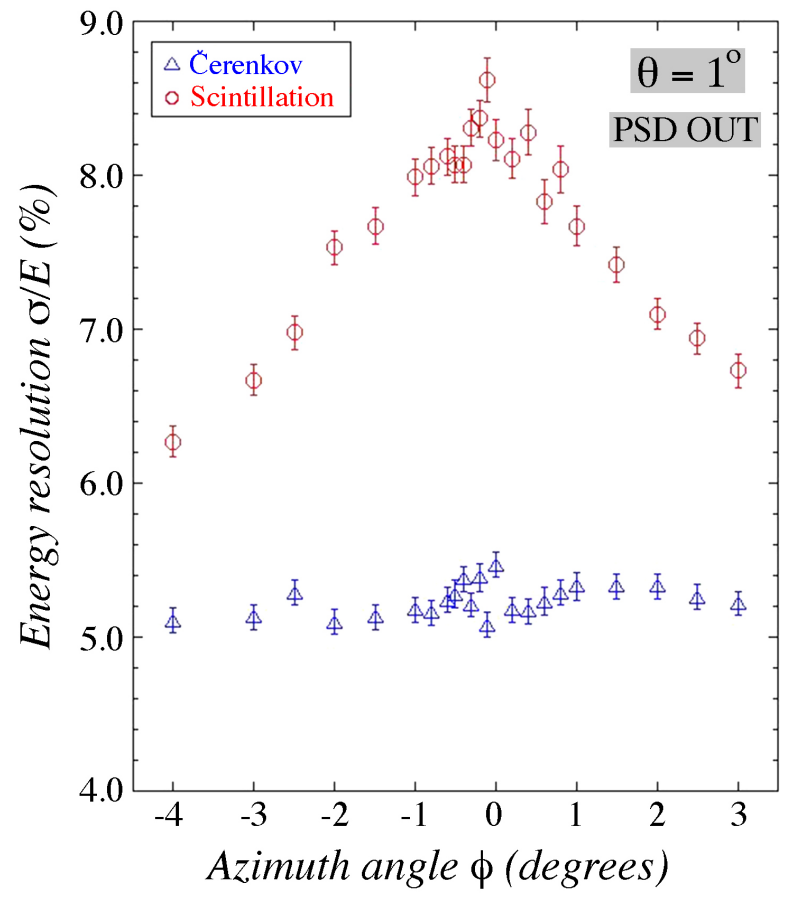

Fig. 6. The energy resolution measured for $20 \mathrm{GeV}$ electrons in the scintillation and the Črenkov channels, as a function of the azimuth angle of incidence $(\phi)$ of the beam particles. The tilt angle $\theta$ was $1^{\circ}$, and the PSD was out of the beam line.

stage of the shower development. Therefore, the calorimeter signal (from this early shower component) depends crucially on the impact point of the particles, if these enter the calorimeter parallel to the fibers. This dependence is quickly reduced when the particles enter the calorimeter at a small angle with the fibers. For example, at $2^{\circ}$ the lateral displacement over a depth of $10 \mathrm{~cm}$ already amounts to 3.5 $\mathrm{mm}$, comparable with the fiber-to-fiber distance. As the angle increases, this early collimated shower component is thus sampled more and more in the same way as the rest of the shower. However, at angles where this is not the case, this effect adds an additional component to the em energy resolution. This effect is, in first approximation ${ }^{8}$, energy independent and thus acts as a constant term.

Now, why does this phenomenon only affect the resolution measured with the scintillation signals? The reason is that the collimated early shower component does not contribute to the Čerenkov signals, since the Čerenkov light produced by shower particles traveling in the same direction as the fibers falls outside the numerical aperture of the fibers. Another spectacular consequence of this phenomenon is the fact that calorimeters of this type can distinguish event-by-event between the energy loss in the form of ionization and radiation when detecting muons, since the Čerenkov fibers only produce signals for the bremsstrahlung component [13]. For

8 The depth of the shower maximum increases with the shower energy, but this is a logarithmic effect. For example, the shower maximum for $10 \mathrm{GeV}$ electrons in copper is located at $\sim 5 X_{0}$, and for $100 \mathrm{GeV}$ at $\sim 8 X_{0}$. 
the $20 \mathrm{GeV}$ electrons, the Čerenkov fibers thus only register shower particles that travel at relatively large angles with the shower axis $\left(20-60^{\circ}\right)$, and such particles are for all practical purposes only found beyond the shower maximum, where the shower is wide compared to the typical distance separating neighboring fibers of the same type. The "constant" term that affects the scintillation resolution is thus practically absent for the Čerenkov signals.

\subsubsection{Effects of the PSD and the tilt angle}

The results measured for a tilt angle $\theta=2^{\circ}$ confirm the above explanation. This is illustrated in Figure 7, where the energy resolution is shown as a function of the azimuth angle $\phi$, for the measurements performed at $\theta=1^{\circ}$ and at $\theta=2^{\circ}$. The deterioration of the energy resolution for small angles $\phi$ is considerably smaller for the $\theta=2^{\circ}$ data, because it is the total angle (i.e., a combination of $\phi$ and $\theta$ ) that counts in this context. As expected, the energy resolution measured for the Čerenkov signals is not affected by the change of the angle of incidence of the beam particles.

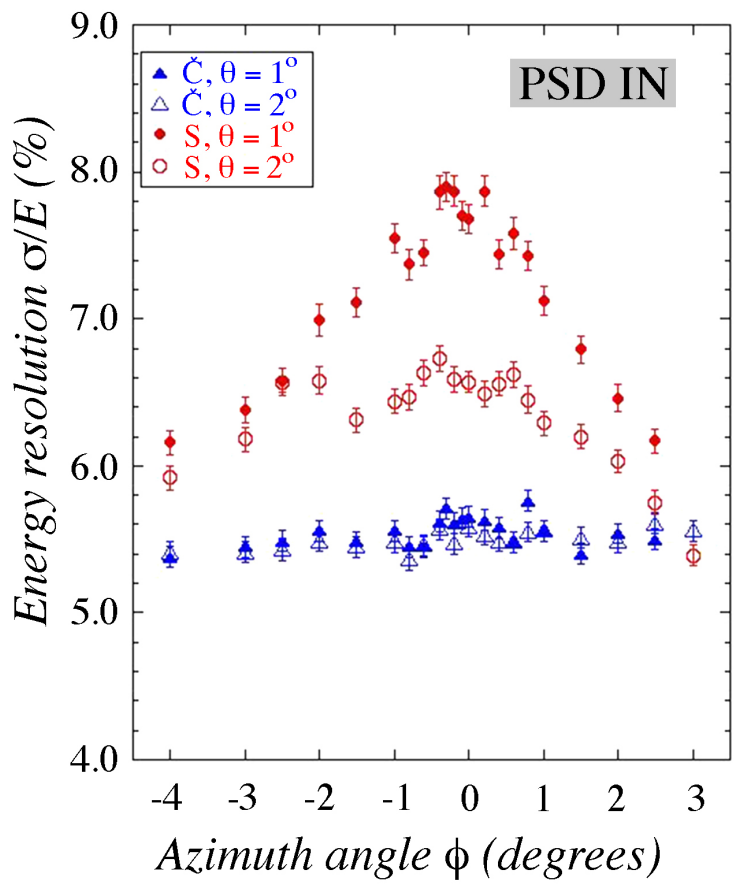

Fig. 7. The energy resolution measured for $20 \mathrm{GeV}$ electrons in the scintillation and the Črenkov channel, as a function of the azimuth angle of incidence $(\phi)$ of the beam particles. Results are compared for tilt angles $\theta$ of $1^{\circ}$ and $2^{\circ}$. These results were obtained with the PSD in the beam line.

However, a careful comparison of the Čerenkov results shown in Figures 6 and 7 does reveal some differences. These are due to the fact that the PSD was in the beam line in one case (Figure 7) and out in the other (Figure 6). We investigated the effects of the PSD absorber on the measured energy resolutions in a systematic way. 
The results of this analysis are shown in Figure 8. It turns out that opposing effects are observed for the energy resolution measured with the two types of signals.

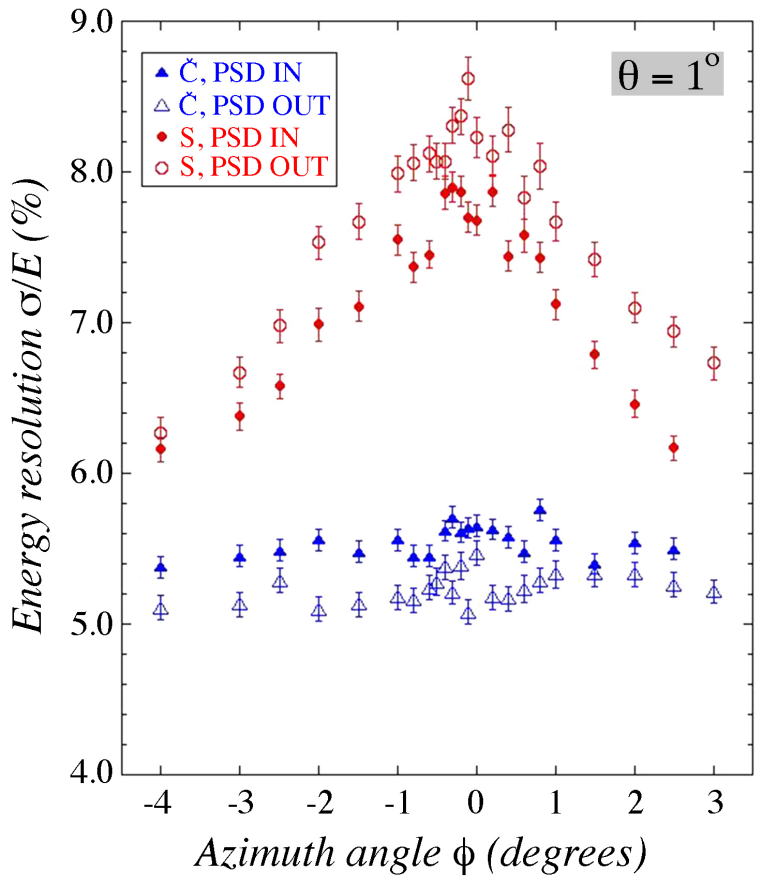

Fig. 8. The energy resolution measured for $20 \mathrm{GeV}$ electrons in the scintillation and Cerenkov channels, as a function of the azimuth angle of incidence $(\phi)$ of the beam particles. Results are compared for data taken with and without the preshower detector in the beam line. The tilt angle $\theta$ was $1^{\circ}$ in both cases.

The energy resolution measured with the Čerenkov signals deteriorates when the PSD is in the beam line. This is to be expected, since the fluctuations that determine the energy resolution of the calorimeter are now increased because of the additional fluctuations in the amount of energy absorbed by this upstream device. Surprisingly, the energy resolution measured with the scintillation signals exhibits the opposite effect, it improves as a result of installing the $1 X_{0}$ thick absorber upstream of the calorimeter. This effect is a consequence of the fact that the absorber causes the shower to start effectively $80 \mathrm{~cm}$ upstream of the calorimeter proper. As a result, upon entering the calorimeter, the shower is somewhat less radially collimated than when the beam particles enter the calorimeter undisturbed. Therefore, the impact point dependence of the calorimeter response is somewhat reduced, and it is this impact point dependence that is responsible for the fact that the scintillation resolution is so much worse for the scintillation signals than for the Cerenkov ones, at very small angles of incidence.

The effect of the PSD absorber on the measured energy resolution is summarized in Table 2, for both signals and for both tilt angles used in these experiments. The table shows that the energy resolution measured with the Čerenkov signals gets worse by $\sim 7 \%$, while the resolution measured with the scintillation signals improves by a 
Table 2

The effect of the upstream (PSD) absorber on the energy resolution of the calorimeter for $20 \mathrm{GeV}$ electrons. Shown is the effect averaged over 21 azimuth angles of incidence $\phi$, ranging from $-4^{\circ}$ to $+4^{\circ}$. The change in the fractional energy resolution (expressed as a percentage of the average calorimeter signal) is positive if the resolution gets worse (i.e., increases) when the PSD is inserted in the beam line. A negative effect indicates that the energy resolution gets better (i.e., decreases) as a result of this operation.

\begin{tabular}{|lcc|}
\hline Signal type & Tilt angle $\theta$ & Change in fractional energy resolution \\
\hline Čerenkov & $1^{\circ}$ & $(+6.0 \pm 0.7) \%$ \\
Scintillation & $1^{\circ}$ & $(-6.8 \pm 1.3) \%$ \\
Čerenkov & $2^{\circ}$ & $(+8.3 \pm 0.5) \%$ \\
Scintillation & $2^{\circ}$ & $(0 \pm 1.3) \%$ \\
\hline
\end{tabular}

similar percentage for a tilt angle $\theta=1^{\circ}$. When the tilt angle is increased to $2^{\circ}$, there is no net change in the scintillation resolution. Since the effective angle of incidence of the beam particles is now larger, the effects that either deteriorate the resolution (by means of fluctuations in the energy deposited in the calorimeter) or improve it (by means of increasing the shower width) apparently cancel each other in this case.

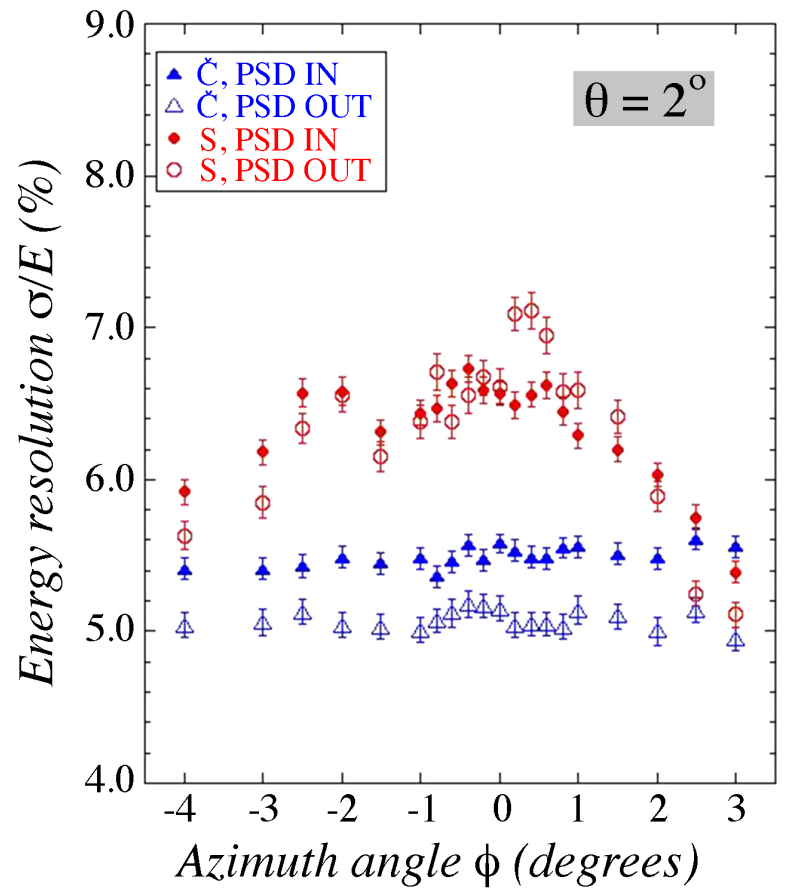

Fig. 9. The energy resolution measured for $20 \mathrm{GeV}$ electrons in the scintillation and Cerenkov channels, as a function of the azimuth angle of incidence $(\phi)$ of the beam particles. Results are compared for data taken with and without the preshower detector in the beam line. The tilt angle $\theta$ was $2^{\circ}$ in both cases.

This is illustrated by Figure 9, which shows the energy resolution for the two types 
of signals as a function of the azimuth angle of incidence, for data taken with and without the PSD in the beam line, at a tilt angle $\theta=2^{\circ}$.

\subsection{Combining the two types of signals}

The use of two independent signals in dual-readout calorimeters is inspired by the beneficial effects of measuring the em shower component event by event for the hadronic calorimeter performance. For the detection of em showers, initiated by electrons or photons, there is absolutely no compelling reason to consider these signals separately. Both types of fibers sample the em showers independently and, as illustrated by the results shown in Figures 6 and 7, the Čerenkov signals provide an even better energy resolution for small angles of incidence, despite the considerably smaller light yield. There is thus absolutely no reason why one should not use the combination of both signals to measure the properties of the showering electrons or photons. Since the sampling fraction and the sampling frequency are twice as large as for the two individual channels, one should expect the contribution of sampling fluctuations to the energy resolution to be reduced by as much as a factor of $\sqrt{2}{ }^{9}$. In addition, the much smaller effective fiber-to-fiber distance should reduce the angular dependence of the energy resolution.

Figure 10 shows the energy resolution for the $20 \mathrm{GeV}$ electron showers for the sum of the scintillation and the Čerenkov signals, as a function of the azimuth angle of incidence $\phi$. For comparison, the results measured for the two signals separately are shown as well. The results qualitatively confirm the naive expectation formulated above. Using the sum of both signals, the energy resolution is found to be better than for the individual signals, and less dependent on the angle of incidence than the resolution measured with the scintillation signals. This is good news, since it means that the dual-readout fiber calorimeter is not only an excellent hadron calorimeter, but also provides good performance for em showers, even at the very small angles of incidence studied in this paper. In compensating calorimeters such as the ZEUS [16] and SPACAL [17] ones, the em energy resolution was limited by sampling fluctuations, which were relatively large because of the requirement of a small sampling fraction. This requirement does not apply for our calorimeter, where the sampling fluctuations (and thus the em energy resolution) can be made much smaller.

The em energy resolution for each of the two signals is determined by sampling fluctuations and by fluctuations in the number of photoelectrons. Apart from these stochastic fluctuations, there is also an angle dependent constant term which only

9 Sampling fluctuations are determined by the number of different shower particles $(N)$ that contribute to the calorimeter signal. If this number doubles, then the statistical precision of this number $(\sqrt{N} / N=1 / \sqrt{N})$, and thus the energy resolution, improve by a factor $\sqrt{2}$. See Reference [11] for more details. 


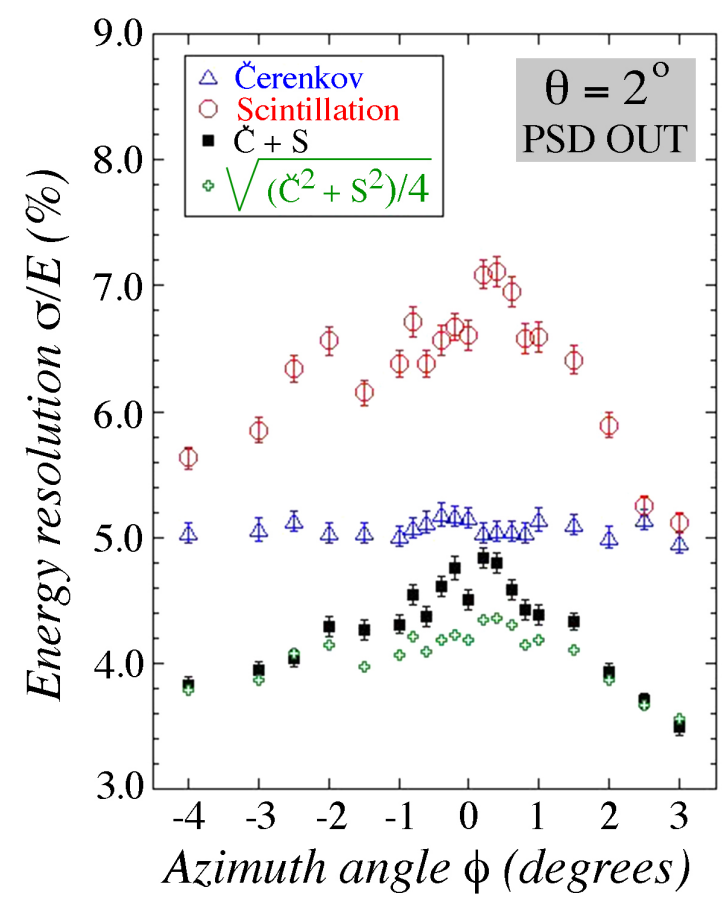

Fig. 10. The energy resolution measured for $20 \mathrm{GeV}$ electrons in the scintillation and the Cerenkov channels and for the sum of both signals, as a function of the azimuth angle of incidence $\phi$. The (green) crosses represent Equation 1 . The tilt angle $\theta$ was $2^{\circ}$ and the PSD was out of the beam line.

contributes to the scintillation resolution. Because of the structure of the calorimeter, the sampling fluctuations are the same for both types of signals, and if these were the only contribution to the energy resolution, then one would expect the resolution to be smaller by a factor of $\sqrt{2}$ for the combined signal. If photoelectron statistics would only affect the Čerenkov resolution, and the constant term only the scintillation resolution, one can show that the resolution for the combined signals is equal to

$$
\frac{\sigma_{[C+S] / 2}}{E}=\frac{\sqrt{\left(\sigma_{C} / E\right)^{2}+\left(\sigma_{S} / E\right)^{2}}}{2}
$$

The (green) crosses in Figure 10 represent the result of this calculation. They describe the measured resolution very well for angles $\phi \geq 2^{\circ}$.

The fact that the energy resolution in the Čerenkov channel is 5\% leads immediately to the conclusion that this signal must consist of at least 400 photoelectrons (20 Cpe/GeV). If we include the contributions of sampling fluctuations, which may be estimated at $2.8 \%$ (see Section 4), we find a Čerenkov light yield of $30 \mathrm{Cpe} / \mathrm{GeV}$ (i.e., 600 photoelectrons for $20 \mathrm{GeV}$ showers). Sampling fluctuations also contribute $2.8 \%$ to the energy resolution for the scintillation channel. Therefore, the constant term deriving from the impact point dependence of the response clearly dominates this resolution, for all angles of incidence studied here. However, by combining the Čerenkov and scintillation signals, the value of this constant term drops below $2 \%$ for angles of incidence larger than 2 degrees. This is consistent 


\footnotetext{
${ }^{10}$ Relevant details about the GEANT4 Monte Carlo simulations performed in the context of the studies described in this paper can be found in Reference [10].
} The angular energy resolution measured with the scintillation signals exhibits an asymmetry, in the sense that the resolution is better for positive values of $\phi$ than for the corresponding negative ones. The same phenomenon is observed for the $\theta=$ $2^{c} i r c$ in Figure 7, where the PSD was installed in the beam line. The asymmetry is much smaller at $\theta=1^{\circ}$ (Figures 7,8 ). We assume that the mentioned asymmetry is caused by the fact that different fibers contribute to the signals and the fiber-tofiber response differences are not necessarily the same at relatively large positive and negative angles. The impact point dependence of the response that is the topic of our study should be symmetric with the angle of incidence. However, as the angle increases, the impact point dependence gradually vanishes, while the other mentioned effect increases and gets its chance to affect the results.

\subsection{Monte Carlo simulations}

One of the reasons for performing the studies described in this paper ${ }^{10}$ was the prediction by Monte Carlo simulations of some very specific effects that would occur for very small angles of incidence [10]. These predictions included:

(1) A dip in the calorimeter response when the angle of incidence $\theta=\phi$.

(2) An electron resolution for the sum of the scintillation and Čerenkov signals that is significantly better, by more than a factor of $\sqrt{2}$, than the resolution measured for either of the two types of signals individually.

(3) An anti-correlation between the signals measured with the scintillating fibers and the signals measured with the Čerenkov fibers.

The first effect would be due to the fact that when $\theta=\phi$, the shower axis may penetrate over some depth inside the calorimeter without encountering a single fiber. In Figure 11, the experimentally measured response is shown together with the GEANT4 prediction, as a function of the azimuth angle of incidence $\phi$, for a tilt angle $\theta=1^{\circ}$. Neither the experimental data nor the Monte Carlo simulations show any evidence for a dip at $\theta=\phi=1^{\circ}$. No dip was observed for $\theta=\phi=2^{\circ}$ either. It should be pointed out that the calculations decribed in [10] concerned a calorimeter with the same structure as the current one, but with lead as absorber material, instead of copper. Whereas lead and copper have approximately the same Moliere radius, the radiation length in copper is larger than that in lead by about a factor of three. While the showers in these two calorimeters thus have approximately the same lateral dimensions, they penetrate much deeper into the copper structure. The probability that the early, collimated shower part is less efficiently sampled for angles of incidence $\theta=\phi$ is thus smaller in copper. 


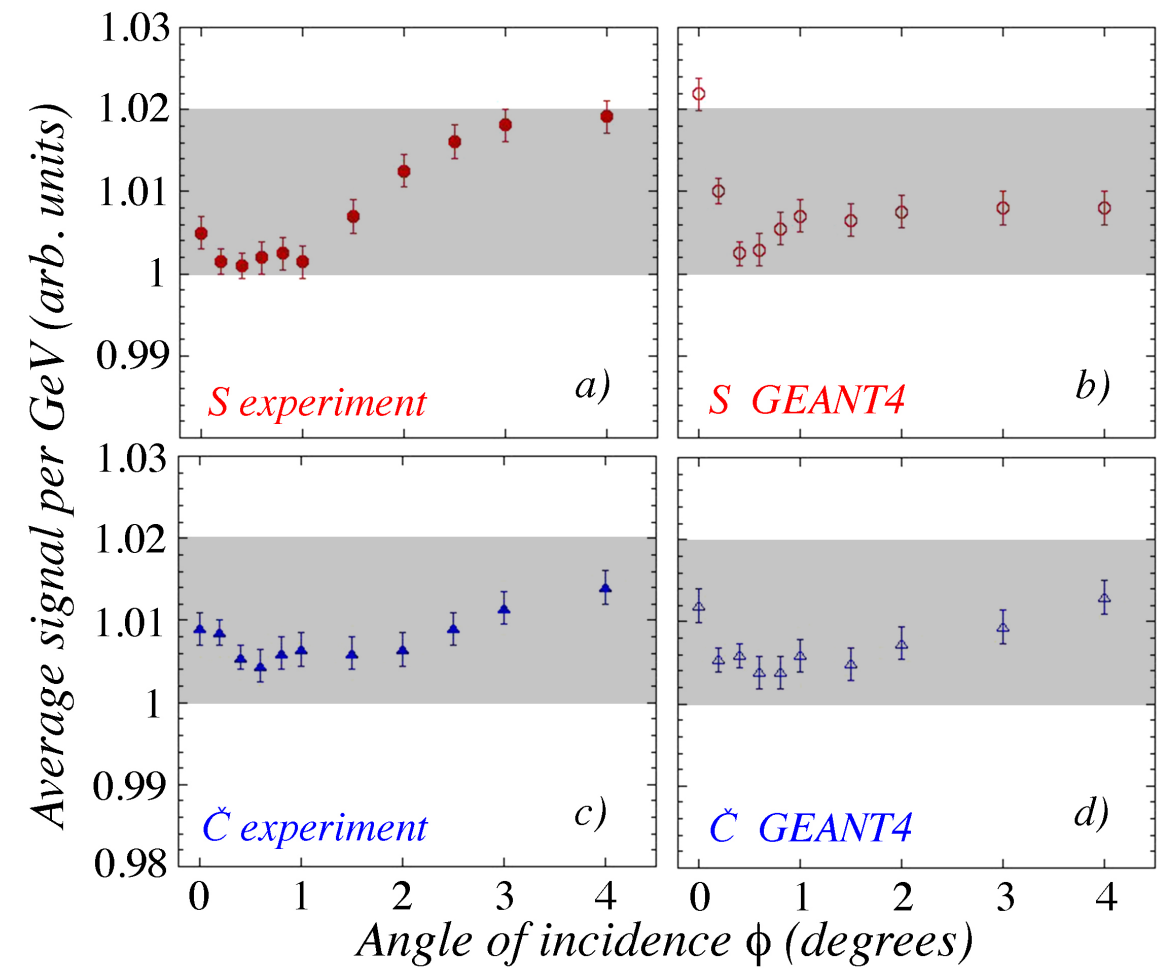

Fig. 11. Comparison between the measured calorimeter response $(a, c)$ and the GEANT4 prediction $(b, d)$, as a function of the azimuth angle $\phi$, for a tilt angle $\theta=1^{\circ}$. Results are given separately for the scintillation $(a, b)$ and Čerenkov $(c, d)$ signals, with the PSD out of the beam line. In all four diagrams, the grey areas represent response values that differ less than $1 \%$ from the average value.

The calorimeter response does exhibit some dependence on the angle of incidence, but the variations are small, typically less than $1 \%$ of the average value (represented by the grey areas in Figure 11). The Monte Carlo simulations describe these variations better in the case of the Čerenkov signals than for the scintillation ones.

The position dependence of the scintillation signal that is the cause of the angular dependence of the scintillation resolution (Figure 6) implies a larger signal when the beam particle enters the calorimeter inside a scintillating fiber than at a position in between two scintillating fibers. Effects 2 and 3 listed above are closely related, since the GEANT4 simulations predict a similar effect for the Čerenkov signals. In other words, when the scintillation signal reaches its maximum value, the Čerenkov signal is at its minimum. This anti-correlation between the two types of signals is visible in the scatter plot shown in Figure 12(b,d). It is also an important factor for the improvement in the energy resolution when both signals are combined (Figure 13).

However, the predicted anti-correlation is not confirmed by the measurements. The experimental data shown in Figures 12(a,c) and 13 are clearly at variance with the GEANT4 results. The absence of an anti-correlation between the two experimental signals should not really be a surprise, since the strong angular dependence of the 

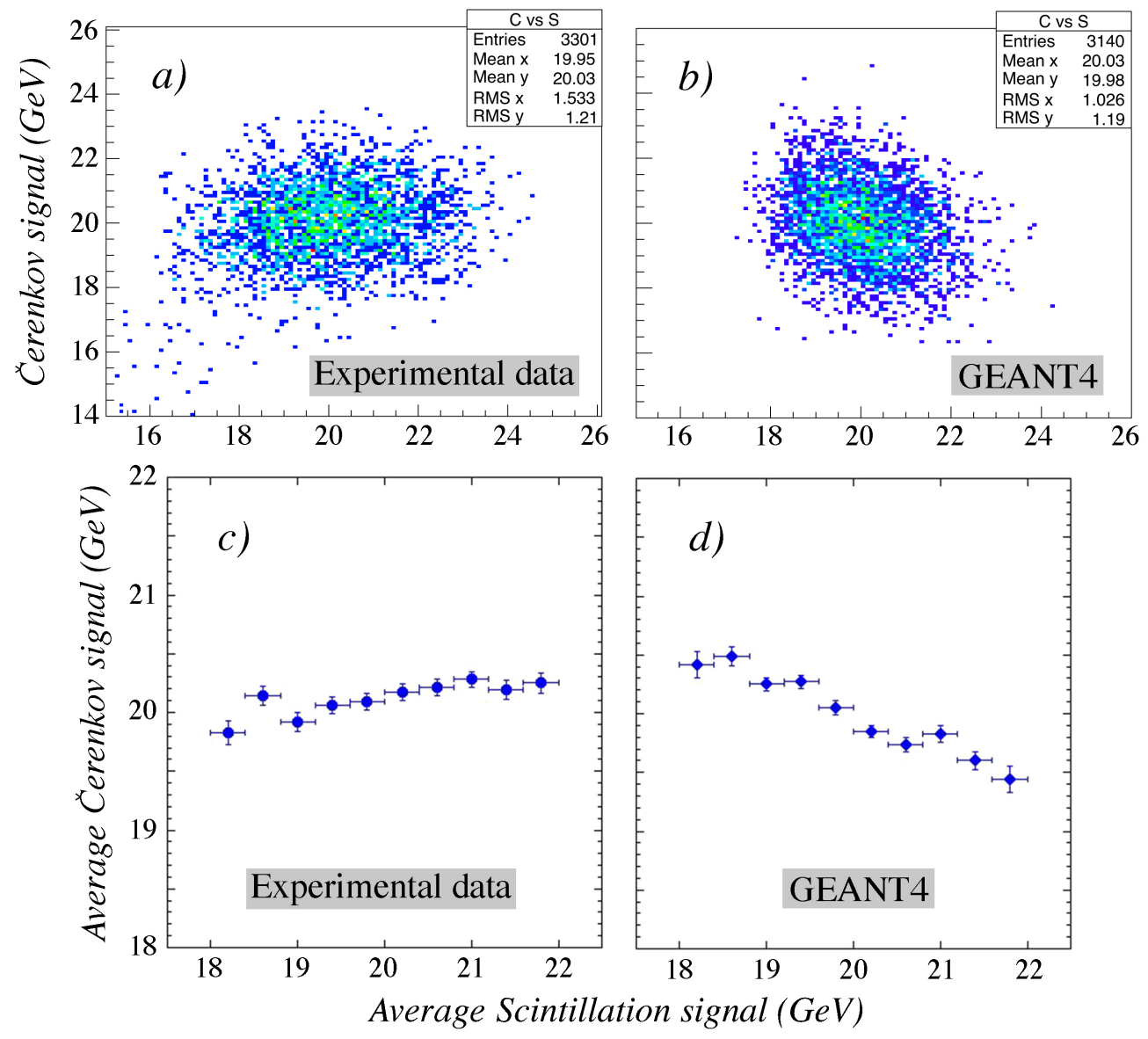

Fig. 12. Scatter plots of the Čerenkov versus the scintillation signals for $20 \mathrm{GeV}$ electrons in the RD52 copper dual-readout calorimeter. Experimental data $(a)$ are compared with GEANT4 simulations $(b)$. Also shown are the average Čerenkov signals as a function of the average scintillation signal, measured in slices with a width of $0.4 \mathrm{GeV}(c, d)$. The angle of incidence of the beam particles was $\left(\phi=1.5^{\circ}, \theta=1.0^{\circ}\right)$.

calorimeter resolution observed for the scintillation signals was found to be absent for the Čerenkov signals (Figures 6 - 9). Since this angular dependence is a direct consequence of the impact point dependence of the calorimeter response, there is thus no reason for an anti-correlation between the two signals.

In Figure 13, the measured energy resolutions are compared with those predicted by the GEANT4 simulations. Results are given separately for the scintillation signals (Figure 13a,b), the Čerenkov signals (Figure 13c,d) and the sum of both signals (Figure 13e,f), for a tilt angle $\theta=1^{\circ}$. The simulated energy resolution for the scintillation signals also exhibits a substantial dependence on the angle of incidence, but the resolution values are considerably better than the experimental ones. In addition, there is a significant increase in the resolution for $\phi=\theta=1^{\circ}$, an effect that was not observed in the experimental data. 


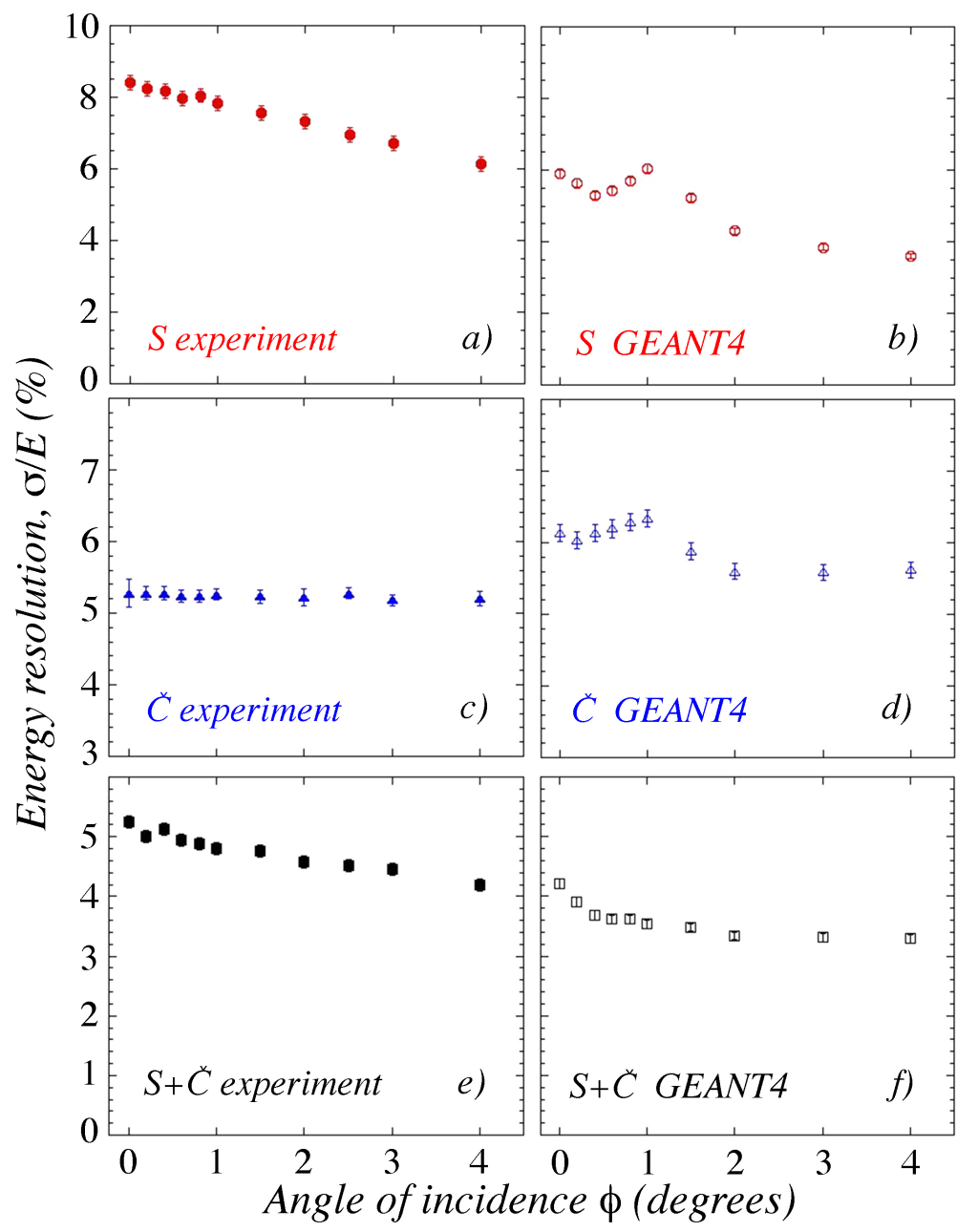

Fig. 13. Comparison between the measured energy resolution for $20 \mathrm{GeV}$ electrons of the RD52 calorimeter and the GEANT4 prediction, as a function of the azimuth angle $\phi$, for a tilt angle $\theta=1^{\circ}$. Results are given separately for the scintillation and Čerenkov signals, and for the sum of both signals. The error bars are statistical only.

The energy resolution in the Crenkov channel also exhibits a maximum value around $\phi=\theta=1^{\circ}$, but the angular dependence is clearly smaller than in the scintillation case. We also notice that the simulated energy resolution is significantly worse than the measured value, for all angles considered here. In addition, the measured energy resolution is independent of the angle of incidence.

The resolutions for the sum of both signals are most similar. The resolution deteriorates slightly when the angle of incidence approaches zero degrees, but the effects are considerably smaller than for the scintillation signal alone. Since the resolution is overestimated in one case and underestimated in the other, the simulated resolution for the sum of the signals is in rather good agreement with the experimental value, ranging from $3-4 \%$ in the simulations and $4-5 \%$ experimentally. 
One may wonder what causes the differences between the experimental and simulated characteristics of the performance of our calorimeter in detecting $20 \mathrm{GeV}$ electron showers. We believe that the intricate calorimeter structure may be an important factor. The calorimeter consists of several different materials (copper, polystyrene, PMMA, air) separated by one millimeter or less, and this represents a very demanding environment for shower simulations. Moreover, the showers are initially extremely collimated, with lateral dimensions that are even small compared to the fiber-to-fiber distance. As a result, the calorimeter (scintillation) signal depends sensitively on the precise impact point of the beam particles. The beam spot is chosen to be $8 \times 8 \mathrm{~mm}^{2}$. This area contains on average 15 fibers of each type, but depending on its precise location and the angle of incidence it may also be effectively 14 in one case and 16 in another. This may lead to significant effects on the response and energy resolution. Experimentally, we don't have information on the precise structure of the beam spot, at the relevant (sub-)mm level. These factors make a detailed comparison between the experimental and simulated results a challenging proposition.

\section{Discussion}

\subsection{High resolution calorimetry}

The results of the studies presented in this paper, combined with the Monte Carlo simulations of the hadronic performance of a full-scale calorimeter of the type tested here [14,15], complete the information needed to come to a total assessment of the experimental performance of the copper based dual-readout fiber calorimeter relative to that of "traditional" compensating calorimeters such as the ones used by the ZEUS experiment [16] and built in the context of the SPACAL project [17]. The latter calorimeters did provide excellent hadronic energy resolution $(\sim 35 \% / \sqrt{E})$, but suffered from two drawbacks:

(1) Because of the use of high- $Z$ absorber material, depleted uranium in the case of ZEUS, lead in the case of SPACAL, the $e /$ mip ratio of these calorimeters was about 0.6 (ZEUS) or 0.7 (SPACAL). This means that the calorimeter response to (em or hadronic) showers was only $60-70 \%$ as large as that of particles that deposited their entire energy in the form of ionization of the absorber material. This phenomenon adversely affected the resolution for jets, where a significant fraction of the energy is carried by soft hadrons that range out rather than develop showers. The fluctuations in the fraction of the jet energy carried by such particles contribute to the energy resolution and thus make it worse than for single hadrons.

(2) To achieve compensation, these calorimeters needed to have a very specific sampling fraction for charged particles, such as to achieve the right response to the many neutrons produced in the hadronic shower development. This sampling fraction was small, e.g., 2.4\% in the case of SPACAL. As a result, the 
em energy resolution was not very good in these calorimeters, since that resolution is typically dominated by sampling fluctuations. For $20 \mathrm{GeV}$ electrons, both SPACAL and ZEUS reported an em energy resolution in excess of $4 \%$.

These effects are strongly mitigated in the calorimeter that is the topic of this paper. Even though the calorimeters we have built so far are too small to fully contain hadron showers, Monte Carlo simulations suggest that hadronic energy resolutions of $30 \% / \sqrt{E}$ should be attainable in a sufficiently large detector $[14,15]$. Since a relatively low- $Z$ absorber is used, the effects of fluctuations in the jet fraction carried by non-showering hadrons should be much smaller than in calorimeters based on high- $Z$ absorber material. The $e /$ mip ratio of copper is 0.85 , i.e., much closer to the ideal value (1.0) than for structures based on uranium as absorber material.

Concerning the electromagnetic performance, the RD52 calorimeter offers the advantage that the sampling fraction is not constrained by the compensation requirement. Our data show that, even in the very unfavorable condition when electrons or photons enter the calorimeter at very small angles of incidence, resolutions better than those reported by ZEUS and SPACAL are obtained in some cases (e.g., 3.4\% at $\phi=3^{\circ}$, Figure 10). At larger angles of incidence, further improvement may be expected, since the impact point dependence of the scintillation signals vanishes. According to the standard formula describing the contributions of sampling fluctuations ${ }^{11}$ to the em energy resolution [11], such fluctuations limit this resolution to $8.3 \% / \sqrt{E}$ in the calorimeter used for the present studies, i.e., $\sim 2.0 \%$ for 20 $\mathrm{GeV}$ electrons. Fluctuations in the number of Čerenkov photoelectrons increase this limit to $\sim 2.8 \%$.

\subsection{The tricky effects of $C / S$ cuts}

The studies performed in the context of this paper taught us one valuable lesson, worthy of sharing with the readership. As described in Section 2.3, the $20 \mathrm{GeV}$ particle beam used for our studies contained a substantial fraction of pions and muons. These particles could be effectively removed from the event samples by means of the external detectors (preshower detector, tail catcher and muon counter). However, in measurements without an upstream absorber (i.e., the PSD was out of the beam line), it turned out to be challenging to completely eliminate the pion contamination.

In a previous study [9], we found that the Čerenkov/scintillation calorimeter signal ratio was an efficient tool to discriminate between electrons and pions. Therefore, we tried to clean up our electron event samples by means of a cut on that signal ratio. Figure 14 shows that this worked quite well. The pion contamination, visible

${ }^{11}$ The dependence of the sampling term on the sampling fraction and the sampling frequency is well described by $a_{\text {samp }}=2.7 \% \sqrt{d / f_{\text {samp }}}$, where the fiber diameter $d$ and the sampling fraction for mips $f_{\text {samp }}$ are $1 \mathrm{~mm}$ and $9.2 \%$, respectively [11]. 


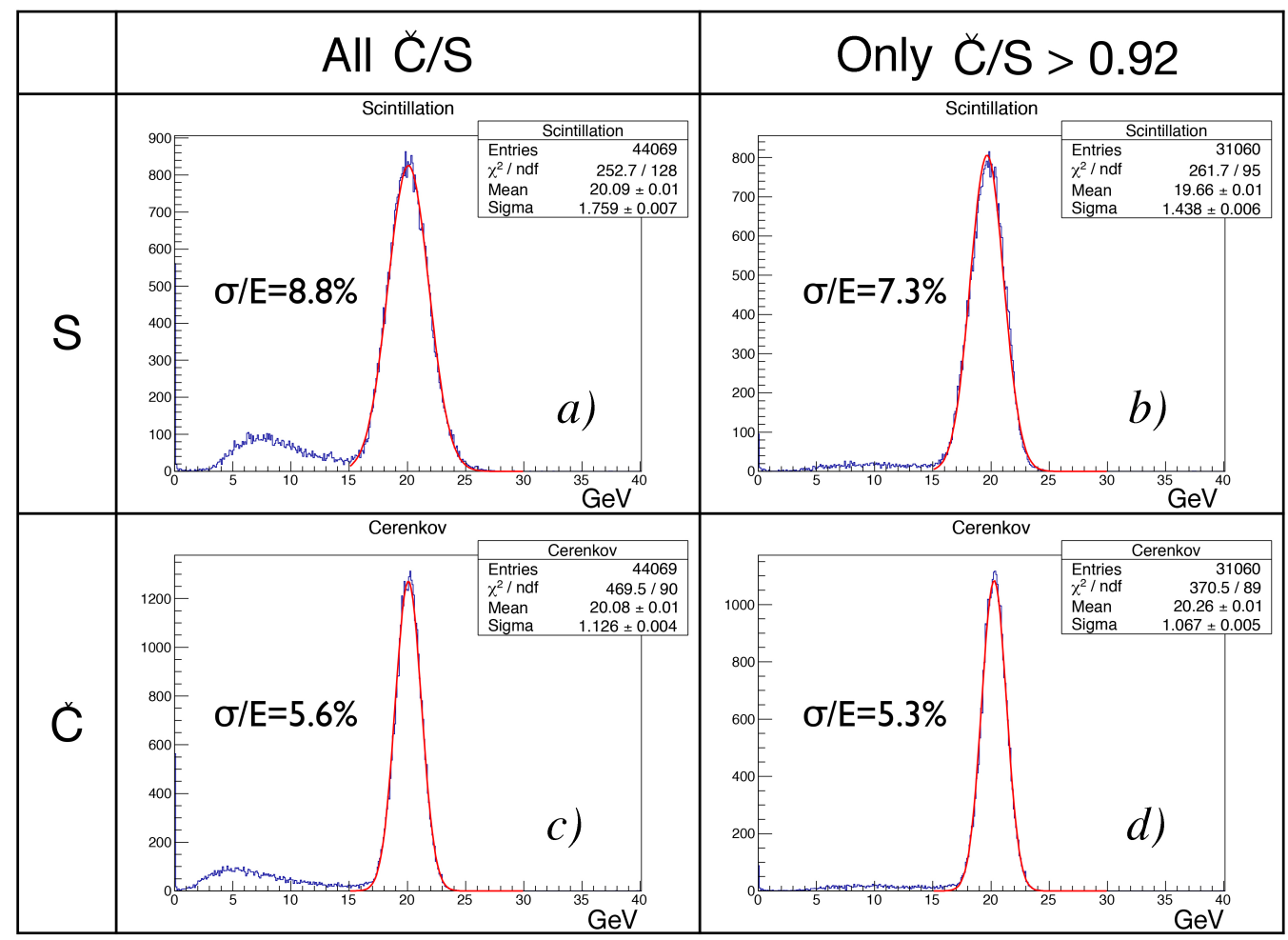

Fig. 14. Signal distributions for the Čerenkov and scintillation signals with and without a cut on the ratio of these two signals. The angle of incidence of the $20 \mathrm{GeV}$ electrons was $\left(\phi=0 \pm 0.5^{\circ}, \theta=1^{\circ}\right)$. The preshower detector was out of the beam line. See text for more details.

as a broad bump below $15 \mathrm{GeV}$, was reduced by a factor of five retaining only events with $C / S>0.92$, while the contents of the electron peak were about $20 \%$ smaller as a result of this cut. However, this figure shows one other amazing effect, namely the improvement in the energy resolution for the electrons, from $8.8 \%$ to $7.3 \%$ in the scintillation signal. These data concern the measurements at a tilt angle of $1^{\circ}$ and an azimuth angle $\phi=0 \pm 0.5^{\circ}$, in other words the configuration for which the effects of the impact point dependence of the scintillation energy resolution reaches its maximum (see Figure 8). And the observed improvement as a result of this $C / S$ cut is even larger than that resulting from inserting the PSD absorber in the beam line. What is going on here?

By retaining events with a large $C / S$ signal ratio, one selectively eliminates events with a large scintillation signal. These are predominantly events in which the beam particles entered the calorimeter close to a scintillating fiber. In this procedure, the effect of the impact point dependence on the energy resolution is diminished, and therefore the measured energy resolution improves, especially in the scintillation channel, where this dependence plays a key role.

Additional support for this explanation may be derived from Figure 15, which shows the calorimeter response to $20 \mathrm{GeV}$ electrons as a function of the azimuth 


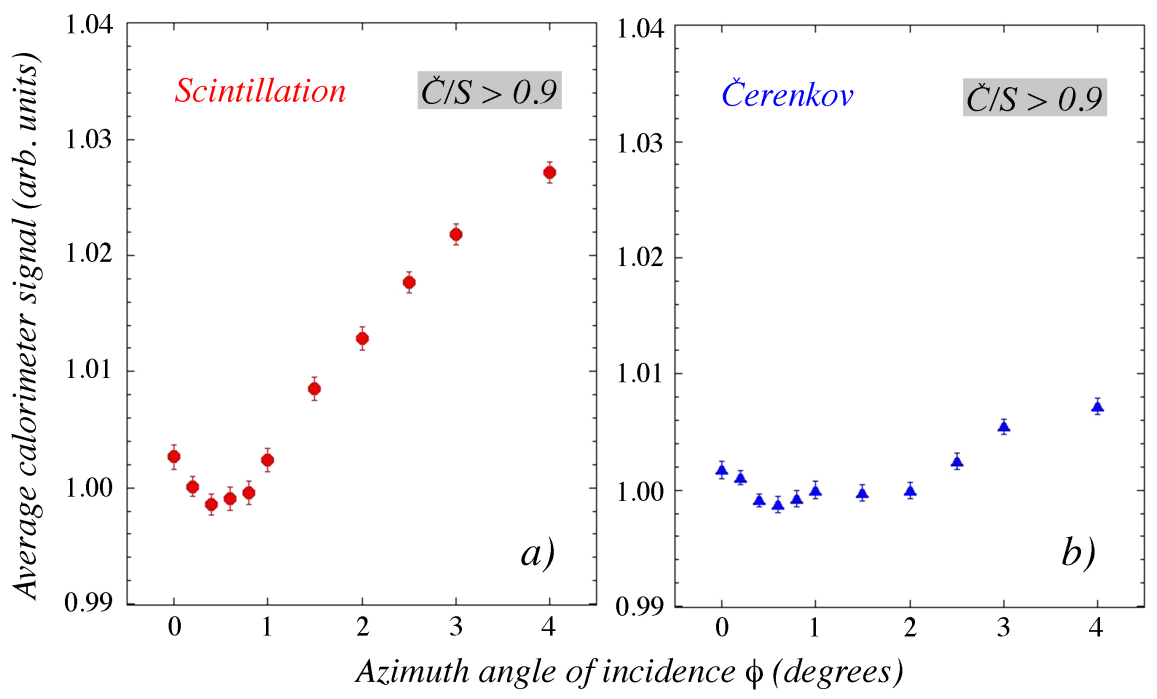

Fig. 15. The average calorimeter signal for $20 \mathrm{GeV}$ electrons in the scintillation $(a)$ and Črenkov $(b)$ channels, as a function of the azimuth angle of incidence $(\phi)$ of the beam particles. The tilt angle $\theta$ was $1^{\circ}$ and the preshower detector was out of the beam line. Only events with a Čerenkov/scintillation signal ratio larger than 0.9 are included. The error bars only include statistical effects.

angle of incidence, $\phi$, for events selected with a cut on the $C / S$ calorimeter signal ratio. As was demonstrated experimentally in reference [18] and confirmed by GEANT4 simulations [10], the scintillation response is larger when the electrons enter the calorimeter inside a scintillating fiber rather than in between scintillating fibers, especially at very small angles of incidence. The selective elimination of events, resulting from the $C / S$ cut, increases with the impact point dependence of the $S$ signals and is thus largest for angles at which the particles enter the calorimeter parallel to the fibers. This is what is observed in Figure 15a.

This experience constitutes an example of the importance of one of the fundamental rules of testing calorimeters: If one wants to measure the performance of a calorimeter, one should only use external detectors to select events (in this case to eliminate pions from the mix), not the calorimeter signals themselves, since this might lead to biased event samples.

\section{Acknowledgments}

We thank CERN for making good particle beams available to our experiments in the $\mathrm{H} 8$ beam. This study was carried out with financial support of the United States Department of Energy, under contract DE-FG02-12ER41783, by Italy's Istituto Nazionale di Fisica Nucleare and Ministero dell'Istruzione, dell'Università e della Ricerca, and by the Basic Science Research Program of the National Research Foundation of Korea (NRF), funded by the Ministry of Science, ICT \& 
Future Planning under contract 2015R1C1A1A02036477. In addition, we thank Korea University for the support received by their researchers who contributed to this project.

\section{References}

[1] E. Eskut et al., Nucl. Instr. and Meth. A401 (1997) 7.

[2] A. Antonelli et al., Nucl. Instr. and Meth. A354 (1995) 352.

[3] S.J. Alvsvåg et al., Nucl. Instr. and Meth. A290 (1990) 320.

[4] M. Beck et al., Nucl. Instr. and Meth. A381 (1996) 330.

[5] G. Bayatian et al., Eur. Phys. J. C53 (2008) 139.

[6] R. Wigmans, New Journal of Physics 10 (2008) 025003.

[7] R. Wigmans, in Handbook of Particle Detection and Imaging, eds. C. Grupen and I. Buvat, vol. 1, 497-517, Springer Verlag (2011).

[8] N. Akchurin et al., Nucl. Instr. and Meth. A735 (2014) 130.

[9] N. Akchurin et al., Nucl. Instr. and Meth. A735 (2014) 120.

[10] N. Akchurin et al., Nucl. Instr. and Meth. A762 (2014) 100.

[11] R. Wigmans, Calorimetry, Energy Measurement in Particle Physics, International Series of Monographs on Physics, Vol. 107, Oxford University Press (2000).

[12] N. Akchurin et al., Nucl. Instr. and Meth. A548 (2005) 336.

[13] N. Akchurin et al., Nucl. Instr. and Meth. A533 (2004) 305.

[14] R. Wigmans et al., RD52 Status Report 2015: Dual-Readout Calorimetry for HighQuality Energy Measurements, Internal document CERN-SPSC-2015-015, CERN, Genève, Switzerland.

[15] R. Wigmans, New Results from the RD52 Project, Proc. of the 13th Pisa Meeting on "Frontier Physics for Frontier Detectors", May 24-30 2015, La Biodola, Elba (Italy), accepted for publication in Nucl. Instr. and Meth.

[16] G. Drews et al., Nucl. Instr. and Meth. A290 (1990) 335.

[17] D. Acosta et al., Nucl. Instr. and Meth. A308 (1991) 481.

[18] N. Akchurin et al., Nucl. Instr. and Meth. A536 (2005) 29. 